\title{
Estrategias didácticas y pensamiento crítico en edad prescolar
}

\author{
Marleni del Aguila Allaín \\ marlemancora@gmail.com \\ https://orcid.org/0000-0003-4283-2748 \\ Universidad César Vallejo, Piura-Perú \\ César Balladares Atoche \\ cesarballadares@hotmail.com \\ https://orcid.org/0000-0001-8242-7742 \\ Universidad César Vallejo, Piura-Perú \\ Fabrizia del Aguila Allaín de Rojas \\ fadelaguila@gmail.com \\ https://orcid.org/0000-0001-9694-0192 \\ Universidad César Vallejo, Piura-Perú
}

\section{RESUMEN}

Organizaciones internacionales, como la UNESCO y UNICEF, buscan mejorar y obtener una educación de calidad, promoviendo un pensamiento crítico reflexivo, donde los estudiantes planteen alternativas de solución ante cualquier situación problemática que atraviesa la sociedad en que se desenvuelven. surge al observar los resultados obtenidos en la prueba de desempeño docente, realizada por el MINEDU(2017-2018) donde señala que en la rúbrica promueve en el estudiante su creatividad, su razonamiento, así como el pensamiento crítico, es donde mayor dificultad obtuvieron las docentes. Ante esta situación se plantea como objetivo proponer una capacitación basada en estrategias didácticas que permita mejorar el pensamiento crítico en estudiantes de pre escolar; con enfoque cuantitativo, tipo aplicada, diseño no experimental y nivel descriptivo propositivo. se utilizó la técnica de la encuesta y el cuestionario como instrumento, la población de 167 y muestra 55 docentes. La conclusión predominante es que las docentes no aplican plenamente habilidades que conlleven a promover el logro del pensamiento crítico en sus estudiantes, tal como lo demuestra la tabla 1 y figura 01 , desde la percepción docente un $64 \%$ está en el nivel medio, seguido de un $36 \%$ en nivel bajo; observando que realizan experiencias de aprendizaje repetitivas y memorísticas.

Palabras clave: pensamiento crítico; estrategia didáctica; docentes del nivel inicial. 


\title{
Didactic strategies and critical thinking in preschool age
}

\begin{abstract}
International organizations, such as UNESCO and UNICEF, seek to improve and obtain a quality education, promoting a reflective critical thinking, where students propose alternative solutions to any problematic situation that the society goes through in which they develop. It emerges when observing the results obtained in the teaching performance test, carried out by the MINEDU(2017-2018 )where it indicates that in the rubric it is promoted in the student their creativity, their reasoning, as well as their critical thinking, is where the greatest difficulty the teachers obtained; Faced with this situation, the objective is to propose a training based on didactic strategies that allows improving critical thinking at the preschool students; with a quantitative approach, applied type, nonexperimental design and purposeful descriptive level. The survey technique and the questionnaire were used as an instrument, the population of 167 and sample 55 teachers. The predominant conclusion is that teachers do not fully apply skills that lead to promoting the achievement of critical thinking in their students, as shown in table 1 and figure 01 , from the teacher's perception $64 \%$ are at the medium level, followed by $36 \%$ at low level; observing that they carry out repetitive and rote learning experiences.
\end{abstract}

Keywords: critical thinking, didactic strategy; initial level teachers.

Artículo recibido: 30 noviembre. 2021 Aceptado para publicación: 29 diciembre 2021 Correspondencia: marlemancora@gmail.com Conflictos de Interés: Ninguna que declarar 


\section{INTRODUCCIÓN}

En Santiago de Chile, la UNESCO(2019) realizó un análisis curricular a nivel regional con la participación de 19 países; señalando una matriz curricular del nivel primaria, en lo que respecta a la lectura para el $3^{\circ}$ y $6^{\circ}$ grado; de los países que participaron, cuyos resultados señalan, que sólo el $46 \%$ de estudiantes en $3^{\circ}$ grado de primaria y $42 \%$ en $6^{\circ}$ grado, tienen dominio en la reflexión y evaluación sobre los textos, lo cual es una habilidad propia para el pensamiento crítico, este tipo de pensamiento es requerida para enfrentar la presencia de información no tan confiable que ha surgido durante el siglo $\mathrm{XXI}$, se requiere comprender lo que se lee, analizar los sucesos que surgen en el entorno y sobre todo buscar dar solución a los múltiples problemas. (Informe Regional de Santiago, 2020)

Por otro lado, el Ministerio de educación (MINEDU) en los años 2017-2018, realiza una evaluación de desempeño a las docentes del nivel inicial, en todo el Perú, los resultados que demostraron con esta evaluación indican que fue en la rúbrica "Promueve en el estudiante su creatividad, su razonamiento, así como el pensamiento crítico", en donde mayor dificultad obtuvieron las docentes, el 51 \% se encuentra en el nivel "suficiente", esto quiere decir que al menos en una ocasión, las profesoras promueve de una manera efectiva las habilidades de pensamiento,

En Tumbes, los resultados de EDD- 2018, arrojó que de las 269 docentes que fueron evaluadas, solo el 23\% logró un nivel destacado, el 53\% en suficiente y el 19\% en el nivel de proceso, y en región Tumbes(2018), provincia de Zarumilla, lugar donde se llevó a cabo la presente investigación, los resultados fueron, de 42 docentes evaluadas, sólo un 9.5\% está en el nivel destacado, un $54.7 \%$ en el nivel suficiente, el $30.9 \%$ en proceso y un $4.7 \%$ obtuvo un nivel muy deficiente, en resumen, el $35 \%$ de las docentes evaluadas en la provincia de Zarumilla, están en nivel en proceso y muy deficiente. Evaluación de desempeño docente Región Tumbes(2018)

Esta investigación tiene una justificación teórica pues pretende contribuir a un conocimiento específico desde una propuesta, describiendo hechos, dichos resultados podrán generalizarse en instituciones de entornos similares. Así mismo podrá llenar algunos vacíos existentes respecto a las variables que se estudian; en cuanto a la justificación práctica, la propuesta presentada, permite resolver un problema de conocimiento sobre el uso de estrategias para mejorar el pensamiento en los estudiantes. 
Este estudio, tiene una justificación social de gran trascendencia al beneficiar a las docentes de este nivel educativo en su desempeño, permitiendo logros satisfactorios en sus estrategias de enseñanza para obtener aprendizajes de calidad en sus estudiantes; será conveniente porque busca demostrar cuanto dominan los docentes estas habilidades cognitivas propias del pensamiento y lograr aplicar la mayor cantidad de estrategias adecuadas para promoverlo.

En el presente trabajo se presentan los resultados que permitió conocer hasta qué punto las docentes aplican estrategias pertinentes para desarrollar este tipo de pensamiento; está desarrollada con el objetivo de proponer un taller de capacitación basado en estrategias didácticas que permita mejorar el pensamiento crítico en el nivel inicial.

\section{ESTRATEGIAS METODOLÓGICAS}

Se llevó acabo un enfoque cuantitativo, implementando técnicas y estadísticas para el análisis e interpretación de los datos recogidos, para ello se elaboró tablas y figuras. Kerlinger (2002) citado por Sánchez (2019), con un diseño no experimental, de nivel transversal descriptivo, por lo que solo se describe lo que se ha investigado (Hernández, et al, 2014).

Tiene carácter propositivo; para Ondé (2019) El diseño no experimental es aquel que solamente se recoge información acerca del fenómeno objeto de estudio, sin intervención o manipulación por parte del investigador, y sin asignación aleatoria. (p.10)

Este diseño permite identificar características de la variable observada; en este caso la variable pensamiento crítico, se obtuvo un diagnóstico, resultado de un cuestionario, donde los resultados arrojaron que existe una falencia en promover el pensamiento crítico, ante ello, se plantea una propuesta de brindar un taller de capacitación a docentes con estrategias que fueron tomadas de las diferentes investigaciones que han tenido la misma problemática, dentro del ámbito nacional e internacional. Tuvo una población de 167 docentes, seleccionando 55 maestras según López (2004) Se obtuvo mediante el muestreo de tipo no probabilístico; por conveniencia de la autora( Hernández, et al, 2014)

Los datos obtenidos del cuestionario, fueron procesados en el programa Excel, los mismos que fueron tabulados por cada una de las dimensiones, tales como habilidades de indagación, nivel del pensamiento inferencial y nivel de pensamiento crítico reflexivo; luego fueron llevados a tablas de frecuencias con porcentajes simples, presentando estos 
resultados en tablas y figuras con sus interpretaciones correspondientes, para ello se empleó diversos equipos tecnológicos tales como laptop y el programa estadístico SPSS. Cabe resaltar que el cuestionario presentó 29 ítems, presentando las opciones : 1= nunca; $2=$ a veces; 3 = siempre; fue validado con la opinión del juicio de 5 expertos. Las docentes del nivel inicial o pre escolar, respondieron cada una de estas preguntas que permitieron, conocer que el $64 \%$ es decir a veces y el 36\% nunca emplean estrategias para promover el pensamiento crítico; según la tabla 1 y figura 01 lo demuestra.

\section{3.- RESULTADOS Y DISCUSIÓN}

\section{Tabla 1}

Nivel de Pensamiento Crítico

\begin{tabular}{ccc}
\hline Nivel & Fcia. & Porcentaje \% \\
\hline Alto & 0 & 0 \\
Medio & 35 & 64 \\
Bajo & 20 & 36 \\
TOTAL & $\mathbf{5 5}$ & $\mathbf{1 0 0}$ \\
\hline
\end{tabular}

Fuente: Cuestionario aplicado a la muestra.

\section{Figura 01}

Nivel de la variable: Pensamiento crítico.

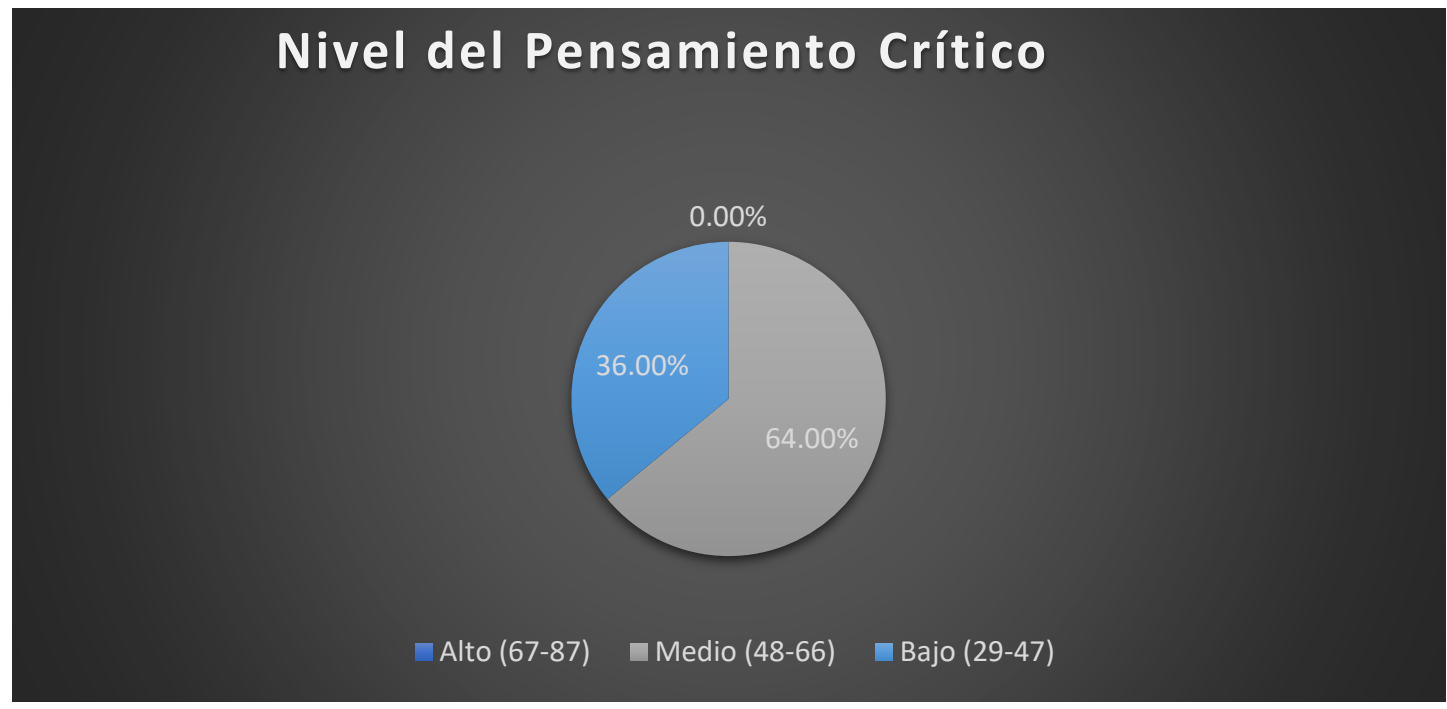

\section{Interpretación:}


En la tabla 1 y figura 01, se aprecia el nivel de la variable pensamiento crítico, desde la percepción docente en el que el mayor nivel es el medio con un 64\% (35 docentes) seguido del nivel bajo con 36\% (20 docentes) calificó en el nivel medio y en el nivel bajo. Como se aprecia en la tabla 1 y figura 01, se aprecia el nivel de la variable pensamiento crítico, desde la percepción docente en el que el mayor nivel es el medio con un 64\% (35 docentes) seguido del nivel bajo con 36\% (20 docentes), dejando de lado el nivel alto, este resultado, deduce que las maestras del nivel inicial no aplican plenamente estrategias que promuevan el pensamiento crítico en los estudiantes, presentando actividades con bajo nivel cognitivo. Por ello, se debe de orientar de manera pertinente a las docentes con estrategias que logren este pensamiento en los estudiantes.

Este artículo concuerda con el trabajo de Morales Rivas(2017)quien concluye que, desarrollar un modelo de estrategias didácticas mejoran las capacidades de quienes reciben este tipo de modelos estratégicos; lo manifiesta de la misma manera Báez(2016) quien realizó una investigación en Chile, indicó que las docentes conocen la importancia de promover este pensamiento, pero en sus planificaciones presentan un nivel cognitivo que no alcanzan a desarrollarlo, indicó además que estas habilidades del pensamiento depende de la forma como se enseñe, considerando a la educación formal quien esté a cargo de ella; en este caso son los maestros.

Tal como lo plantea la UNESCO, a través de la oficina Regional de Educación para América Latina de Santiago de Chile, en su informe de julio del 2020, da a conocer los resultados obtenidos del análisis curricular que se dio en América Latina, donde participaron : Argentina, Bolivia, Brasil, Chile, Colombia, Costa Rica, Cuba, Ecuador, El Salvador, Guatemala, Honduras, México, Nicaragua, Panamá, Paraguay, Perú, República Dominicana, Uruguay y Venezuela; donde se planteó ¿Qué se espera que aprendan los estudiantes de América Latina y el Caribe? En el área de lenguaje sus resultados donde menos favorecidos están nuestros estudiantes latinos es la reflexión y evaluación de los textos, los mismos que son necesarios para el desarrollo del pensamiento crítico, Ante ello, se espera que los docentes fomenten diversas estrategias para alcanzar los objetivos trasados.

De la misma manera, apoya lo declarado por Mendoza(2015), quien empleó 30 preguntas a través de un cuestionario, cuyo propósito fue medir las dimensiones del pensamiento en estudiantes universitarios, resaltando que se debe desarrollar competencias investigativas. 
Cabe mencionar que no tan solo se debe desarrollar estas competencias investigativas en el nivel universitario, sino al contrario desde pequeños, esto concuerda con el enfoque de indagación, donde los niños del primer nivel educativo, inician con este tipo de habilidades a través de la exploración, plantean hipótesis, registran y analizan información, estructurando así nuevos conceptos del mundo que los rodea. Programa curricular nivel inicial(2016)

Es importante resaltar lo que plantea y sustenta la teoría Tríadica, una de las teorías en que se basa esta investigación, cuyo autor es Robert Sternberg (1986) quien describe la relación de la inteligencia con tres subteorías que poseen las personas; la primera subteoría componencial, considera al mundo interno de cada persona, con él está el pensamiento analítico y académico, el mismo que le permite investigar, planear y ejecutar; la segunda subteoría experiencial, comprende la relación que existe entre el mundo y el exterior.

Esto quiere decir que determina el manejo de propias experiencias, utiliza para ello el pensamiento creativo, enfatizando cuan original e innovador es y; la tercera subteoría contextual hace referencia a la forma en que la persona se moviliza en su entorno, considerando al pensamiento práctico, adaptable y con éxito; implica tener la habilidad en dar solución a los problemas. Citado por Bezanilla et al ( 2018)

Ante estos resultados de las diferentes investigaciones donde se toman como referencias, se sustentan que los niños y niñas "aprenden de manera progresiva a partir de las oportunidades que se le brinden en el contexto que se desarrollan, con el acompañamiento del adulto"(Inicial,2021 p.12)

\section{CONCLUSIÓN}

Durante el análisis de resultados de la variable pensamiento crítico, se puede concluir que las docentes no están aplicando plenamente habilidades que conlleven a promover el logro del pensamiento crítico en sus estudiantes, tal como lo demuestra la tabla 1 y figura 01, desde la percepción docente un $64 \%$ está en el nivel medio, seguido de un $36 \%$ en nivel bajo.

Esta investigación propone un taller de capacitación docente, donde se les brindará las estrategias pertinentes de cada una de las dimensiones del pensamiento crítico; tales como promover y mejorar las habilidades de indagación, habilidades de inferencia y sobre todo mejorar el nivel de pensamiento crítico. 
El taller se realizará tomando como referencias 5 estrategias didácticas que se les brindará a las docentes que imparten sus enseñanzas en el nivel inicial, permitiendo mejorar en el estudiante el pensamiento crítico. Estas son, la observación, la formulación de preguntas, el planteamiento de hipótesis, el juego de roles y los debates.

Esperamos que las entidades públicas pertinentes, tal como la Ugel Zarumilla, directoras de instituciones del nivel inicial, promuevan la participación de sus docentes a este taller de capacitación denominado "La didáctica que mejorará el pensamiento crítico en el nivel inicial."

\section{LISTA DE REFERENCIAS}

Báez Alcaíno, J. E.(2016) Niveles de concreción del desarrollo de habilidades de pensamiento como contenido fundamental transversal del currículum. [Tesis doctoral, Universidad de Barcelona] https://dialnet.unirioja.es/servlet/tesis?codigo $=137968$

Bezanilla Albisua, María José; Poblete Ruiz, Manuel; Fernández Nogueira, Donna; Arranz Turnes, Sonia; Campo Carrasco, Lucía(2018) El Pensamiento Crítico desde la Perspectiva de los Docentes Universitarios. Revista Estudios $\begin{array}{llll}\text { Pedagógicos } & \text { XLIV }, & \mathrm{N}^{\circ} & \text { 89-113. }\end{array}$ https://scielo.conicyt.cl/pdf/estped/v44n1/0718-0705-estped-44-01-00089.pdf

Evaluación Ordinaria del Desempeño Docente 2018 Informe nacional Nivel InicialTramo

II.MINEDUhttps://evaluaciondocente.perueduca.pe/media/11565198575Informe -Final-EDD-2018-Tramo-II.pdf

Inicial, N. D. E. E. (2021). Mejora de los aprendizajes - 2021 Sesión 1 Enfoque por competencias. 1-19. Eseñar al nivel real de los aprendizajes - i para el nivel inicial. Curso virtual.http://perueduca.

Hernández-Sampieri, R., Fernández-Collado, C. y Baptista-Lucio, P. (2014). Selección

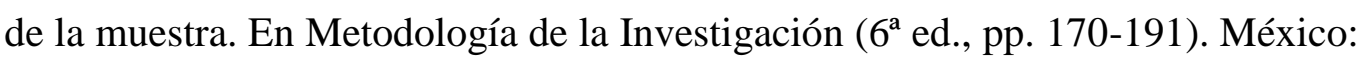
McGraw-

Hill.http://metabase.uaem.mx/xmlui/bitstream/handle/123456789/2776/506_6.p

$\underline{\mathrm{df}}$

López, Pedro Luis. POBLACIÓN MUESTRA Y MUESTREO. Punto Cero [online]. 2004, vol.09, n.08 [citado 2021-12-23], pp.69-74. Disponible en: 
http://www.scielo.org.bo/scielo.php?script=sci_arttext\&pid=S181502762004000 $\underline{100012}$

Mendoza G., P. L. (2015). La investigación y el desarrollo de pensamiento crítico en estudiantes universitarios. [Tesis doctoral, Universidad de Málaga, 1194].Retrieved from http://hdl.handle.net/10630/11883

Morales Rivas, Magaly del Milagro(2017) Modelo de estrategias didácticas con el uso de los tics para desarrollar aprendizajes significativos en el curso de proyecto de investigación e innovación tecnológica del instituto Master System, 2016. [Tesis doctoral, Universidad de César Vallejo, Chiclayo]. https://hdl.handle.net/20.500.12692/18807

Ondé Pérez, Daniel(2019) Revisión del Concepto de Causalidad en el Marco del Análisis Factorial Confirmatorio. Revista Iberoamericana de Diagnóstico y Evaluación - e Avaliação Psicológica. RIDEPhttps://www.aidep.org/sites/default/files/202001/RIDEP54-Art9.pdf

$\begin{array}{lll}\text { Programa } & \text { curricular nivel inicial(2016) }\end{array}$ https://repositorio.minedu.gob.pe/handle/20.500.12799/4548

Sánchez Flores, F. A. (2019). Fundamentos Epistémicos de la Investigación Cualitativa y Cuantitativa: Consensos y Disensos. Revista Digital de Investigación En Docencia Universitaria, 101-122. https://doi.org/10.19083/ridu.2019.644

UNESCO, Santiago.2020. Comunicado Regional lanzamiento y análisis curricular de América Latina el $y$ Caribe p.1-4. https://es.unesco.org/sites/default/files/comunicado-regional-lanzamientoanálisis-curricular

UNESCO. (2019). Resultados del Análisis curricular del Estudio Regional Comparativo y Explicativo (ERCE 2019). Obtenido de https://es.unesco.org/news/resultadosanalisis-curricular.

UNICEF América latina y Caribe(2020) https://www.unicef.org/lac/plan-12pensamiento-critico. 